\title{
AN OPTIMAL ADAPTIVE APPROACH USING BEHAVIORAL PROMISCUOUS CACHING AND STORAGE-CAPACITY CHARACTERISTICS FOR ENERGY CONSERVATION IN ASYMMETRICAL WIRELESS DEVICES
}

\author{
Constandinos X. Mavromoustakis \\ Department of Computer Science, \\ INTERCOLLEGE \\ 46 Makedonitissas Avenue, P.O.Box 24005 \\ 1700 Nicosia, Cyprus \\ mavromoustakis.c@intercollege.ac.cy
}

\author{
Helen D. Karatza \\ Department of Informatics, \\ Aristotle University of Thessaloniki \\ 54124 Thessaloniki, Greece \\ karatza@csd.auth.gr
}

\begin{abstract}
Current approaches for tuning the energy conservation do not take into consideration the storage requirements of each device, the traffic and channel data rate as well as parameters concerning the caching activity for packet forwarding. In this work, an evaluation scheme is proposed based on each node's self-scheduling energy management. This scheme is associating crucial metrics of an ad-hoc wireless host with the remaining capacity units of each device, communicating via asymmetrical wireless links. Promiscuous caching is used in the ad-hoc based connectivity scenario where the-so called- promiscuous caching threshold parameter is introduced in order to bound the associated mobility with storage/capacity and traffic characteristics and figure out a suitable pattern for optimal energy conservation. Through experimental simulation, the proposed energy management scheme is thoroughly evaluated in order to meet the parameters' values where the optimal energy conservation is achieved.
\end{abstract}

\section{INTRODUCTION}

Wireless network's survivability and the offered reliability are entirely based on the remaining energy of each node. One of the major impacts on energy conservation of nodes is the kind of wireless interface that each node communicates with one another. This is somehow based on the traversed traffic on the channels. If nodes have low or moderate traffic load, network interfaces are in the idle state most of the time. Thus turning off idle network interfaces is an efficient way to conserve energy or to significantly reduce the consumed energy. In general, by turning off idle network interfaces we simply reduce the number of nodes that are available for packet routing, resulting in a significant reduction in network performance. Effective energy conservation techniques should maintain a balance between the amount of energy a network conserves and the available resources (spare forwarding capacity) to sustain acceptable network performance. Network partitioning problems may occur if network interfaces are turned off for prolonged time, resulting in unacceptable QoS for end users. Hence, the energy conservation mechanism has to be closely collaborative with the incoming traffic characterization with the routing protocol behavior used by nodes, and with the time constrained behavior of the node being in the idle state. All the above should be considered in the forwarding decision by nodes while maintaining the packet forwarding mechanism.

In many cases the network lifetime could be categorized into different factors that influence performance like the channel metrics knowledge and characterization, fading consequences and approximation techniques [3], as well as real time traffic characterization [4] etc. Thus in this work a new approach using behavioral promiscuous ${ }^{1}$ caching and storage-capacity characteristics is presented for energy conservation in asymmetrical wireless devices. Asymmetry plays a major role in wireless devices since it affects the end to end delay and the offered reliability increasing the energy consumed. Classified packets [5] have some further characteristics (class of service, storage used, time limitation) which in some cases -of prioritized traffic-these characteristics could be an efficient way for saving power in wireless devices. The proposed approach is based on stream's characteristics

\footnotetext{
Promiscuous means that all wireless hosts are able to cache anywhere, at anytime their requested contents.
} 
with respect to different caching behavioral characteristics and storage-capacity characteristics. These characteristics are modeled and through the estimated formula the subsequent metrics of the scheme can be bounded. In order to achieve power conservation, progressively the power is saved from node to node, contributing to overall power savings, which results in significant conservation of the energy in the network. A comparison is performed with respect to different traffic rates [20] following a real time asymmetry channel. It is obvious that the self tuned behavioral promiscuous caching enables significant energy conservation by using different delay metrics based entirely on promiscuous caching and on each channel's characteristics.

The organization of the paper is as follows: Section 2 discusses the related work that has been done on similar energy conservation schemes and the conducted solutions by different schemes. Section 3 then introduces the proposed behavioral promiscuous caching technique with the modeled storage-capacity parameters and shows how these parameters affect the energy conservation of asymmetrical wireless ad-hoc environments. Section 4 provides the evaluation of the proposed scheme and presents the simulation results focusing on the behavioral characteristics of the scheme and the newly introduced parameters, and section 5 concludes with a summary of our contribution and suggestions for further research.

\section{RELATED WORK}

Multiple energy conservation approaches have been proposed for infrastructureless wireless networks that are exploited at either the link layer or network layer protocols or a combination of both. These approaches seem to be inefficient when implemented in real time ad hoc networks due to the temporally random nature of access protocols. The schemes that are based only on protocols where different states are adopted randomly (switching node's states to "low energy"), cause severe degradation of network capacity [2]. There is already a significant amount of research work, which addresses the routing layer [7-10] for enabling energy conservation as well as the MAC and physical layers [11-15] by using different approximation techniques. This work neither involves any layered end-to-end mechanism nor enables any routing layer involvement. The proposed mechanism takes action on a MAC and physical layers and on application layer where the behavioral promiscuous caching is taking place, with respect to storage and capacity characteristics. Many protocols have been designed and use different mechanisms to reduce energy consumption; they are classified into two categories: active and passive protocols. Active techniques conserve energy by performing energy conscious operations, such as transmission scheduling using a directional antenna
[16], and energy-aware routing [17]. The passive techniques conserve energy by scheduling network interface devices to the sleep mode when a node is not currently taking part in any communication activity (packet forwarding).

Currently the majority of recently introduced protocols take into account many external factors (air, location etc), but not the traffic/channel characteristics and users' behavior and storage characteristics. Some of the recently proposed protocols deal with MAC layer [7] issues and network layer [17] issues and some are based on topological and geographical information-based techniques (GAF) [18]. Authors in [18] proposed a scheme based on the division of the entire network into small virtual pieces (grids). This area which is recognizable by geographical information, allows only one node to be active in the grid while the other nodes turn off their interfaces to conserve energy. In [19] the goal is to turn off nodes without significantly diminishing the capacity or connectivity of the network. The connectivity and forwarding capability as stated in [19], is maintained by keeping the nodes that constitute a backbone infrastructure, in active mode and switching off the other. Also a traffic-load history determination in association with battery lifetime has been examined in [4]. In [4] the research is focused on load history characterization for each node, targeting the energy conservation for delay and non-delay sensitive services. The self-similarity of packet traffic characterization studied in [4] allows nodes to change their state depending entirely on their traffic history.

A framework combining the behavioral promiscuous caching characteristics and storage-capacity characteristics in an asymmetric wireless communication network is not yet explored in the energy conservation research. While some approaches based on overhearing or fading techniques do not actually address the association of $E C$ (Energy Conservation) problem with any aspects of traffic, in this work this scenario is considered and examined. Parameters that an EC technique should be aware are user's behavior, data generation, traffic aggregation, means of network control, control mechanisms based on feedback and network connectivity. In this work the channel's traffic is considered and it is combined with stream-traffic characteristics and the associated time that the caching mechanism wastes in order to temporarily save data packets destined for a node. The available capacity is then combined -based on the model approach in [1] with traffic and caching characteristics in order to enable minimization of energy consumption and mitigation of the subsequent parameters. 
The 18th Annual IEEE International Symposium on Personal, Indoor and Mobile Radio Communications (PIMRC'07)

III. AN ADAPTIVE APPROACH USING

BEHAVIORAL PROMISCUOUS CACHING FOR EC BASED ON STREAM ORIENTED DELAY SENSITIVE MECHANISM

This section describes the approach using behavioral promiscuous caching method for EC based on Stream Oriented Delay Sensitive mechanism. This mechanism associates the available capacity and caching characteristics of each node with the traffic characteristics, as well as with the connection characteristics of each device which is asymmetrically connected $^{2}$ with any other device on the network. Overall this method targets the power saving on each device, minimizing the aggravation of the energy consumption of the entire network.

\section{III-(A). SYSTEM MODEL AND LAYERING ISSUES}

Nodes in wireless networks typically rely their survivability on their battery energy. Energy consumption should be minimized in order to save power for -initially- each wireless device independently or all of the WLANs' devices, in a decentralized form. The effects on reducing each node's power consumption has been studied thoroughly [4, 15-18]. However an association of the capacity and caching characteristics and measures with respect to efficient message passing for self-tuning energy consumption is explored in this work. The energy conservation mechanism has to be closely collaborative with the upper layer protocols used, to maintain the packet forwarding mechanism, in an error free mode. A best candidate solution must be costeffective and adaptive, and should be able to keep abreast of any possible changes in the network in terms of load and failures. The above issues combining the unpredictable movements of the users, along with each device's technical characteristics (like asymmetry) should be balanced. This work proposes a way to avoid any technical discrepancies that may exist between different devices, the energy consumption of each device or sudden energy deficiency of all the devices in a certain path.

Figure 1 shows the main architectural communicational stack using the Stream Oriented Delay Sensitive (SODS) mechanism introduced in [1]. The SODS mechanism takes place in Physical, MAC and Application specific layers of the communication stack. Considering the variability of the distance covered by each device, the energy savings should be strictly device dependant. This distance dependant communication can be associated with a particular caching-capacity metrics and can define a relation in power-capacity with respect

\footnotetext{
Most researches focus on symmetric devices' characteristics. This work considers an asymmetric model.
}

to delay in power saving. Figure 1, addresses the combination of SODS mechanism with the effects of caching on each device independently. SODS mechanism can be repeatedly applied while packets are being sent, and in collaboration with the parameterized cached mechanism that will be explained in next section. SODS mechanism runs at each node taking actions for maintenance and urge/instructs the wireless interface/ hardware to sleep and wake up according to the estimated SODS method [1]. Figure 1 shows in shaded form the SODS with promiscuous caching along with the application layer collaboration. These collaborations among these layers are primarily responsible for all parameterized mechanisms explained in section 3.2.

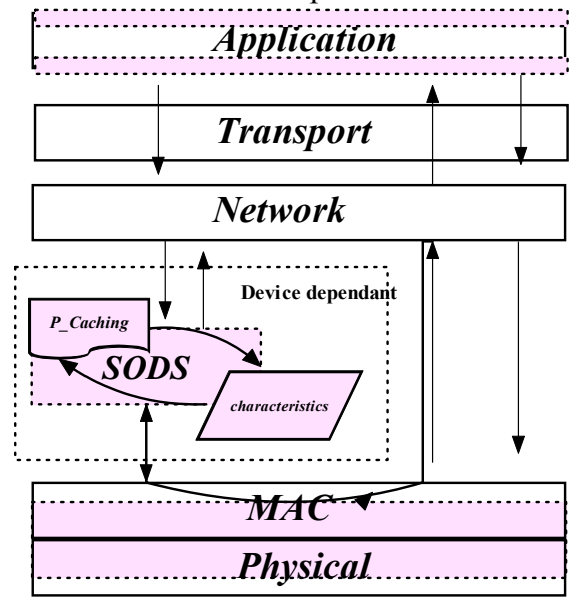

Figure 1: Architectural communicational stack of Stream Oriented Delay Sensitive (SODS) mechanism which takes place in Physical, MAC layer and Application specific layers of the communication stack.

III-(B). MODELING THE MECHANISMS FOR STREAM ORIENTED DELAY SENSITIVE POWER CONSUMPTION

The explosion of traffic over data communications networks has resulted in a growing demand for Quality of Service (QoS) techniques to ensure network reliability, particularly in regard to delay sensitive applications. There are two main traffic types: delaysensitive traffic and loss-sensitive traffic. Delay-sensitive traffic is characterized by rate and duration and may need real-time transmission. Examples include video conferencing, telephone, and audio/video on demand, which usually have stringent delay requirements but can accept a certain loss. Loss-sensitive traffic is characterized by the amount of information transmitted. Examples are Web pages, files, and mail. This enterprise becomes difficult in wireless infrastructureless systems since in such systems there is no guaranteed end-to-end communication. The power control mechanism in collaboration with a traffic manipulation mechanism can prolong battery life by using a minimum required 
transmission power. According to traffic engineering, the energy that is consumed to transmit a data unit is directly proportional to the total energy consumption by a constant number. Thus if a sender wants to transmit a stream of data at rate $\mathrm{R}$ to a receiver, the corresponding transmission power $\mathrm{P}$ can be expressed as:

$$
P=R \cdot d^{r} \text {, where } 2<r<4
$$

In equation (1), $P$ is the consumed power, $r$ is the path loss exponent based on different channel models, $d$ is the distance between to adjacent wireless nodes, and $\mathrm{R}$ is data rate of the channel. Equation (1) assumes symmetrical channels in the communication between devices. In this case according to traffic engineering of symmetric links, power consumption over a long link is much higher than the total power consumption over several short links. Therefore:

$$
\left(d_{1}+d_{2}+d_{3}+\ldots+d_{n}\right)^{r}>>d_{1}^{r}+d_{2}^{r}+d_{3}^{r}+\ldots+d_{n}^{r}(2)
$$

Equation (2) is valid only if we consider symmetric links. Considering asymmetric links between nodes as Figure 2 shows, then the power consumed equals to:

$$
P=\sum_{i=0}^{n} R_{i} \cdot d_{i}^{r}
$$

where $R_{i} \cdot$ is the transmission rate of $i$ link, and $d_{i}^{r}$ is the distance of $i$-node to the next node-hop. It stands that:

$$
d_{1} \neq d_{2} \neq d_{3} \neq d_{4} \ldots \neq d_{n}
$$

By using an exponential notation, we assume that power is reduced progressively $[2,4]$ with the remaining capacity on each node, which is evaluated by:

$$
P_{i_{c}}=P_{i} \cdot e^{e^{C_{i}}}
$$

and

$$
P_{i_{C}}=e^{e^{c_{i}}} \sum_{i=0}^{n} R_{i} \cdot d_{i}
$$

where $\mathrm{C}$ is the total data density. We now need to associate the data caching ability of each node at any time during packet transfer. In order to achieve this we make use of the average caching delay experienced by all the involved mobile peers in forwarding activity as indicated in [23] as follows:

$$
\bar{\delta}^{(m)} \approx \frac{\tau_{0}}{m} \log _{2} i_{P}
$$

where $m$ is the number of identical sized chunks that the file is divided, $i$ is the number of wireless peers in the path $P$ and $\tau_{0}$ is the amount of time taken to download the whole file, if downloaded from a single peer. Therefore a threshold for optimal caching (at any intermediate node) is chosen as:

$$
\sigma_{i}=\frac{\sum_{i=0}^{n} R_{i} \cdot d_{i}}{\bar{\delta}^{(m)}}, \sigma_{i} \cdot \bar{\delta}^{(m)}=\sum_{i=0}^{n} R_{i} \cdot d_{i}
$$

where $\sigma_{i}$. is the promiscuous caching threshold parameter or when minimized is considered as the optimal caching parameter for minimum energy consumption. The parameter $\bar{\delta}^{(m)}$ is the promiscuous caching delay (duration in $\mathrm{sec}$ ).

Due to fading the power of the radio waves decreases with the distance. Fading is one of the main characteristics of a signal's propagation over wireless links. From the aspect of noise and shadowing, fading is not desirable. If we associate the data delay during data revocation from a node with fading characteristics then according to [21], we consider that:

$$
\xi_{i}=\frac{R_{i} \cdot \Phi_{i}}{e^{C_{j}}}
$$

where $C_{j}$ is storage capacity of the node for which data packets from node $i$ are requested.

Thus by encapsulating equation (6), equation (4.2) above can be written:

$$
P_{i}=\sum_{i=0}^{n} R_{i} \cdot d_{i} \cdot e^{e^{c_{i}}}, C_{i}, \xi_{i} \geq 0
$$

where equation (7) above estimates the consumed power from node $I$ to node $n$ over a single asymmetric path. In (7) there is partial association of Energy consumption with capacity and delay characteristics. However these characteristics are not factual since the nature of these characteristics are entirely based on other parameters of the network.

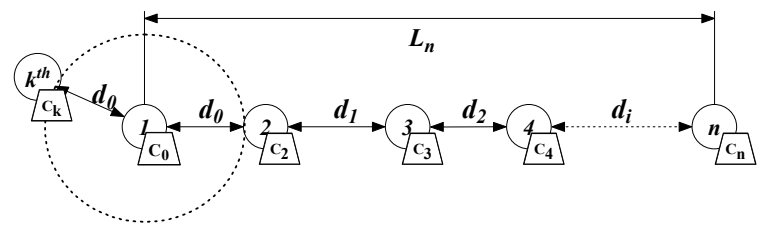

Figure 2: A non-linear communication between adjacent nodes (asymmetric communication) with the corresponding capacities of each wireless host.

Thus, substituting the above equation 5.2 into equation 7, we have:

$$
P_{i}=\sigma_{i} \cdot \bar{\delta}^{(m)} \sum_{i=0}^{n} e^{e^{c_{i}}}, C_{i}, \xi_{i} \geq 0
$$

Using the power estimation above we considered a stream oriented approach as introduced in [1]. Packets are a part of streams which comprise a file -with file chunk correlations (like multimedia audio and video streams). All packets have a time $\tau$ for reaching destination in a free erroneous mode as expressed in [11, 
$20]$ in order to arrive correctly at a specified destination. In our approach we also used the $S_{n}$ notation and streaming specification as used in [1]. $S_{n}$ is considered as the streaming packets parameter of a single application. These packets are marked as prioritized. The $S_{t-\tau, j}\left(S_{1}, S_{2}, S_{3}, \ldots, S_{n}\right)$ is called streaming delay bound, where $\mathrm{j}$ is the number of the possible intermediate nodes, that any of the stream packets $S_{n}$ might follow, and $S_{t-\tau, j}$ is the upper bound of the required time for correct reception of the stream, at the destination. The described scenario uses prioritized and non-prioritized packets (delay sensitive and "don't care" packets [5]). These packets have a bounded time delay $\tau$ to reach any specified destination. In our scheme "don't care" packets are further delayed onto intermediate nodes where prioritized packets are enforced to continue their "journey" to reach sooner their destination.

In a bounded time transmitted packets must be delivered to the requested destination while delaying the "don't care packets" onto intermediate nodes. This delay is estimated by taking measures using the SODS mechanisms explained earlier along with the bounded capacity and the promiscuous caching threshold parameter that characterizes each node and should not exceed a certain value. The estimated delay aggravates the remaining capacity (by hosting packets/reserving more capacity) of each intermediate node that hosts temporarily data packets. Simulation results show that this aggravation trades off the energy consumed on every node separately, though the promiscuous caching enables -instead of total-the partial aggravation of the remaining energy of any individual node. It is therefore considered that after consecutive simulation experiments, overall this scheme saves energy while prolonging network's lifetime. Additionally it offers an in-time arrival of data packets as well as overall throughput optimization. According to $[4,5]$ the cached data packets (information) might not be disastrous for the end-to-end delay, if cached packets are manipulated in a specified time interval. By using the mechanism used in [1] and the same time considering the remaining capacity on each node with the optimal caching parameter being in a determined interval, this methodology can significantly reduce the total consumed energy of all the terminals in the network. In essence it encompasses upper protocol layer independency.

\section{SIMULATION EXPERIMENTS AND DISCUSSION}

To demonstrate the methodology discussed in this paper, we performed exhaustive discrete time simulations of the proposed scenario under several different conditions. The basic goal is to estimate the promiscuous caching threshold parameter and set the energy limitations between energy, capacity and traffic impact and limitations.

$$
\begin{gathered}
\text { IV-(A) . SPECIFICATIONS AND ROUTING } \\
\text { PROTOCOL USED }
\end{gathered}
$$

Considering the need of bandwidth and the limited battery power for wireless devices, it is necessary to apply efficient routing algorithms to create, maintain and repair paths with least possible overhead production [2]. The generated overhead from the route maintenance process, cause significant reduction of network performance, and increase the end-to-end delays and delay variations $[1,4,5,20,23]$. Therefore a protocol which considers the above should be chosen.

In the implementation of the proposed scenario the Zone Routing Protocol (ZRP) [16] is used. ZRP is a hybrid protocol which combines the reactive and proactive modes. The ZRP is considered advantageous because it allows to a certain node to accurately know its neighbors within a zone. These devices should be in a zone (of asymmetrical communication links) that could be accessible in a fixed number of hops. Since ZRP allow the absolute communication with neighbors, is considered less expensive, while neighbors contribute in the routing process. Particularly, ZRP divides the network into several routing zones which are specified by a determined number of hops. This allows the routing protocol to be adjustable for different operational network conditions such as heavy traffic [13-15].The specifications used for simulating our scheme are based theoretically on the WaveLAN PC/Card energy consumption characteristics found in the study by Feeney and Nilsson [22]. By using the WaveLAN $\mathrm{PC} / \mathrm{Card}$ energy consumption characteristics we have approximate reference measures in our simulation scenario.

$$
\begin{array}{ll}
\text { IV-(B) } . ~ & \text { SIMULATION RESULTS OF THE } \\
& \text { PROPOSED SCENARIO }
\end{array}
$$

In our experiments we took into account the $\sigma_{i}$-the promiscuous caching threshold parameter (or bounded optimal caching parameter for minimum energy consumption) and values of $P_{i}$ in terms of $\xi_{i}$, for satisfying $\operatorname{Min}\left(P_{i}, \xi_{i}\right), \operatorname{Max}\left(R_{i}\right), \operatorname{Min}\left(\bar{\delta}^{(m)}\right) . \quad$ The promiscuous caching threshold parameter is estimated, taking into account many factors like end-to-end delay, hop-by-hop latency and power consumed over the delay and capacity measures. Some simulation experiments also were performed using different node capacities in order to evaluate the proposed scenario's response in 
contrast to node's required capacity for maintaining $\operatorname{Min}\left(P_{i}, \xi_{i}\right), \operatorname{Max}\left(R_{i}\right), \operatorname{Min}\left(\bar{\delta}^{(m)}\right)$. These achievements should be established on a hop by hop basis.

As previous researches cogently stated, the cached information destined for a proper node should be stored in a node with higher residual energy. As simulation process shows, if nodes with higher level of residual energy are chosen in the path, then the network partitioning probability $[4,5]$ is further reduced. Therefore the cached process which takes place is chosen on a recursive path basis [1] in order to face discrepancies between packet delays and the storage capabilities of each node. The tree of Node Residual Energy (NRE) is created in order to enable an on demand caching and to assign the certain packets to a certain node of a specified path.

For implementing the described scenario, we used the spine model of [4] (based on C/Objective $\mathrm{C}$ programming language). Topology of a 'grid' based network was modeled according to the grid approach described in [4]. In the simulation of the proposed scenario we used a two-dimensional network, consisting of 50 dense nodes. The topology changes dynamically as well as density and on a non-periodic basis (asynchronously as real time mobile users do). Each link (frequency channel) has max speed of $11 \mathrm{Mb}$ per sec (ideal speed), and the propagation path loss is the tworay model without fading. We have also modeled on each node's communication protocol, an agent for generating and evaluating the data packets that are destined for a proper destination. The network traffic is modeled by generating constant bit rate (CBR) flows. Each source node transmits one 512-bytes ( 4Kbits) packet. Packets are generated at every time step by following Pareto distribution as depicted in [4], and are destined for a random destination which is uniformly selected.

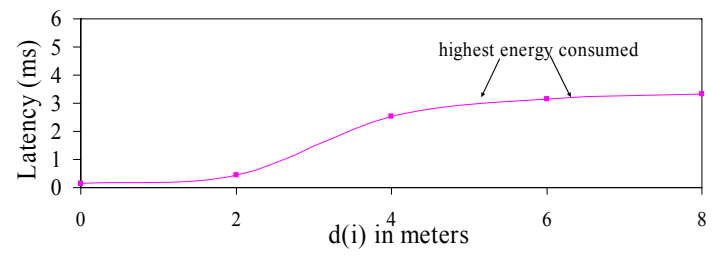

Figure 3(i): Hop by hop latency with the average distance d(i) of each node in meters.

Figure 3(i) shows the hop by hop latency with the average distance d(i) of each node in meters. Figure 3(i) depicts that close range devices are consuming less energy than long range devices. Additionally close range devices are experiencing the minimum possible latency in ms. Figure 3(ii) shows the latency with the reserved storage capacity of each node in GB. It can be easily spotted out that as much storage is reserved then the delay is significantly increased.

Figure 4 shows the mean power consumed with the average distance $\mathrm{d}(\mathrm{i})$ of each node in meters. It is true that for range distances less than 6 the power consumption of each device is kept at low level. On the contrary being higher than $6 \mathrm{~m}$ then it increases dramatically. Figure 5 shows the promiscuous caching threshold parameter with respect to the average caching delay experienced by all the involved mobile peers during the end-to-end forwarding activity. After consecutive conducted experiments through simulation, the promiscuous caching threshold parameter is found to lie in the range of $0.2<\sigma<0.99$. It was also found that if promiscuous caching threshold parameter is kept closer to 1 then the delay is strictly associated with the average caching delay experienced by mobile peers in the end-toend forwarding activity. This principle is illustrated in figure 5 .

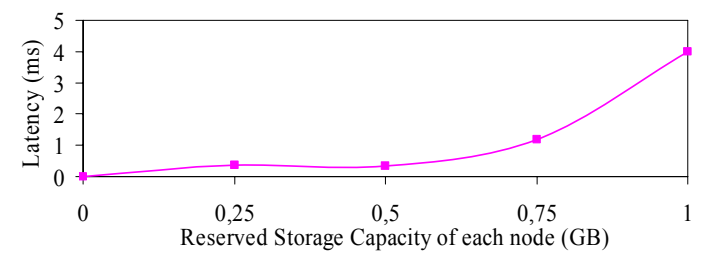

Figure 3(ii): Hop by hop latency with the reserved storage capacity of each node in GB.

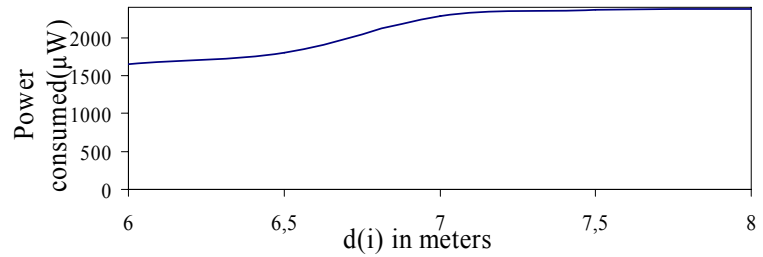

Figure 4: The mean power consumed with the average distance d(i) of each node in meters.

Figure 6 illustrates the mean power consumed with $S_{t-\tau, j}$ which comprises the upper bound of the required time for correct reception of the stream at the destination node. The mean delay should be the minimum for the correct reception of the stream at the destination node. Figure 6 shows that for high power consumption values, the mean delay is significantly higher. That is because according to equations (4.2), (6), (7) and (8) the consumed power, from node $i$ to node $n$ over a single asymmetric path, will significantly increase the power consumed if the delay sensitive data density, is at high levels. As a result the promiscuous caching discussed earlier takes place and in turn the mean power consumed is increased. All the above are aggravated by each node's fading characteristics where, due to fading, the power of the radio waves decreases with the distance, and the power consumed is significantly higher. 


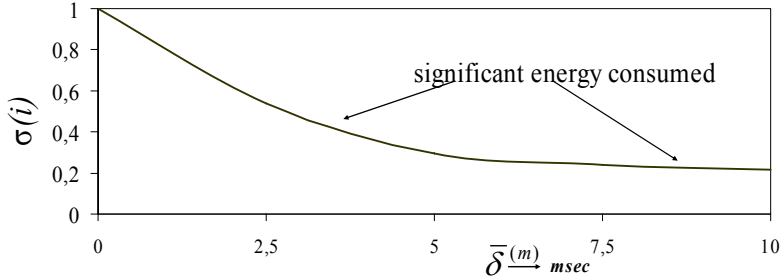

Figure 5: The promiscuous caching threshold parameter with the average caching delay experienced by all the involved mobile peers in the end-to-end forwarding activity.

Figure 7 shows the mean power consumed with the promiscuous caching threshold parameter with different caching and storage measures. Different measures were examined for the capacity and the caching reserved capacity on each device. It is of significant interest to mark out that the optimal combination for enabling energy conservation is the $1 \mathrm{~GB}$ storage capacity and only half the storage capacity is used for caching.

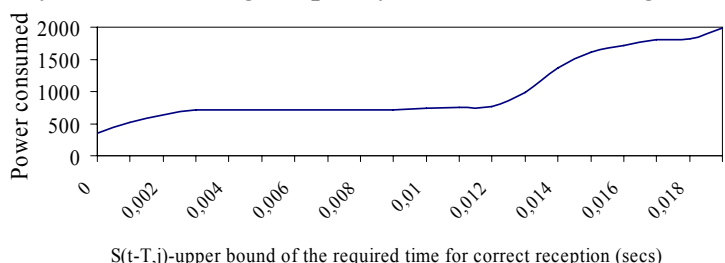

Figure 6: The mean power consumed with the upper bound of the required time for correct reception of the stream at the destination node.

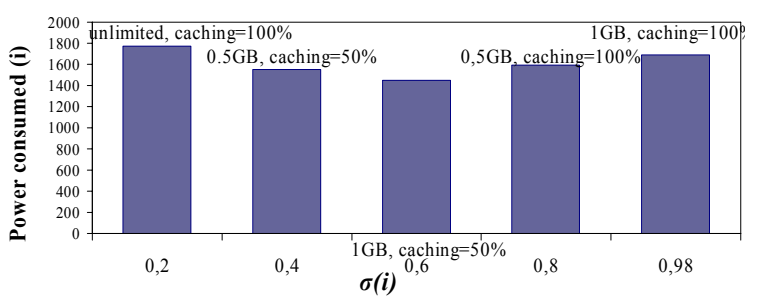

Figure 7: The mean power consumed with the promiscuous caching threshold parameter with different caching and storage measures.

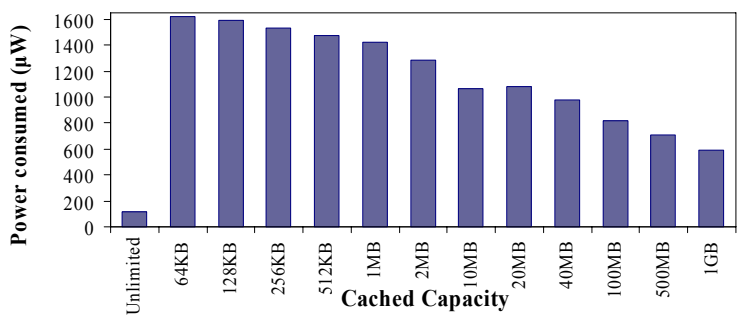

Figure 8: The power consumed with respect to different capacity measures for each node.

Figure 8 shows the power consumed with respect to different capacity measures for each node. Of considerable interest is that the bounded -but highercapacity (1GB) can conserve energy on each node during the caching process. The unlimited capacity can save energy as well.

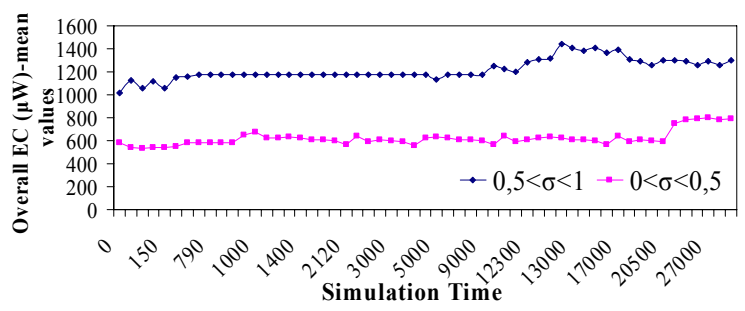

Figure 9: Mean Energy consumption in each network's zone (ZRP-based) at any time during simulation.

In figure 9 the mean Energy Consumption in each network's zone (of the ZRP) is illustrated, at any time during simulation. Figure 9 shows the two different range domains for $\sigma$. The most significant outcome of Figure 9 is that the energy consumed remains at relatively low levels, when $\sigma$ is balanced between values. This denotes that there is association of the exponential nature (equation 7 and 8) of the capacity with node's communication distance range and that the prioritization of delay sensitive packets can efficiently affect the energy conservation mechanism. Overall results show that $P_{i}$ is significantly minimized as well as $\xi_{i}$ for $\operatorname{Min}\left(P_{i}, \xi_{i}\right), \operatorname{Max}\left(R_{i}\right), \operatorname{Min}\left(\bar{\delta}^{(m)}\right)$.

\section{CONCLUSIONS AND FURTHER RESEARCH}

In this paper, we have proposed an association of the power consumption control with some crucial metrics using the promiscuous caching concept. Promiscuous caching threshold parameter is introduced in order to bound of mitigated mobility, storage/capacity and traffic characteristics with the overall energy consumed by the system. Experimental simulation results show that the system under certain conditions offers the optimal energy conservation. The non-linear proposed communication model takes into consideration the transmission power of the adjacent nodes and associates the data delay with capacity measures during data revocation. Also it considers an association with the traffic density, storage measures and the impact of caching promiscuously.

A further examination of such scenarios with thorough modeling will be the subsequent steps in our research. The exponential nature of crucial metrics (i.e. data packet delays, packet loss, interarrival delay variations, different traffic patterns) is always an open ended issue particularly in dynamically changing topologies. 
The 18th Annual IEEE International Symposium on Personal, Indoor and Mobile Radio Communications (PIMRC'07)

\section{REFERENCES}

[1]. C.X. Mavromoustakis and H.D. Karatza, "Performance measures of stream-oriented power consumption for asymmetrical communication in wireless ad-hoc networks". Proceedings of 40th Annual Simulation Symposium (ANSS), IEEE Computer Society Press, SCS, Norfolk, VA, March 25 - 28, 2007, pp.310-317.

[2]. N. Ahmed, M. Khojastepour, B. Aazhang, "Outage Minimization and Optimal Power Control for the Fading Relay Channel", IEEE Information Theory Workshop, Houston, May 2004, pp. 458-462.

[3]. N. Morinaga, R. Kohno, S. Sampel. "Wireless Communication Technologies: New Multimedia Systems", ISBN: 0-792-37900-4, 2002, Kluwer Academic Publishers, New York.

[4]. C. X. Mavromoustakis, H. D. Karatza. "Adaptive Trafficbased Control Method for Energy Conservation in Wireless Devices". Journal of Simulation Practice and Theory (SIMPRA), Elsevier, Volume 13, Issue 3, April 2005, pp 213-232.

[5]. C. X. Mavromoustakis, H. D. Karatza. "Optimized QoS Priority Routing for Service Tunability and Overhead Reduction using Swarm based Active Network Scheme". Journal of Computer Communications, Elsevier, Volume 29, Issue 6, April 2006, pp. 765-780.

[6]. S. Buttery, A. Sago. "Future Applications of Bluetooth”.,BT Technology Journal, Volume 21, 3,2003, Kluwer Academic Publishers, pp.48-55.

[7]. M. Maleki, K. Dantu and M. Pedram. "Power-Aware Source Routing Protocol for Mobile Ad Hoc Networks". Proceedings of International Symposium On Low Power Electronics and Design (ISLPED), pp. 72-75, Aug. 2002.

[8]. S. Singh, M.Woo, C.S. Raghavendra. "Power aware Routing in mobile ad hoc Networks". Proceedings of the 4th annual ACM/IEEE international conference on Mobile computing and networking, pp.181-190, October 1998, Dallas, Texas, United States.

[9]. Y. Xu, J. Heidemann, D. Estrin. "Adaptive energyconserving routing for multihop ad hoc networks", Research Report 527 USC/Information Sciences Institute, October 2000 , pdfurl="http://www.isi.edu/ johnh/PAPERS/Xu00a.pdf".

[10]. C. Toh. "Maximum Battery Life Routing to Support Ubiquitous Mobile Computing in Wireless Ad Hoc Networks", Volume 39, Issue 6, June 2001 pp. 138 - 147 IEEE Communications Magazine.

[11]. W. Ye, J. Heidemann, and D. Estrin. "An energy efficient MAC protocol for wireless sensor networks". Proceedings of the IEEE Infocom, pp. 1567-1576. New York, NY, USA.

[12]. S. Singh, C.S. Raghavendra. "PAMAS: Power Aware Multiaccess Protocol with Signaling for Ad Hoc Networks," ACM Computer Communication Review, vol. 28, no. 3, July 1998.

[13]. R. Kalidindi, L. Ray, R. Kannan, S. S. Iyengar. "Distributed Energy-Aware MAC Protocol for Wireless Sensor Networks". Proceedings of International Conference on Wireless Networks, Las Vegas, Nevada, 1-5 June 2003, pp. 282-286.

[14]. E. S. Jung, N. H. Vaidya. "An Energy Efficient MAC Protocol for Wireless LANs". Proceedings of INFOCOM 2002, vol.3, pp. 1756-1764.

[15]. S. Biswas, S. Datta. "Reducing Overhearing Energy in 802.11 Networks by Low-power Interface Idling,". Proceedings of IEEE International Conference on Performance, Computing, and Communications, 2004, pp. 695- 700 .
[16]. Z. Haas, M. Pearlman. "The performance of query control schemes for the zone routing protocol". ACM/IEEE Transactions on Networking 9(4), 2001, pp. 427-438.

[17]. S. Doshi, T. Brown. "Minimum Energy Routing Schemes for a Wireless Ad Hoc Network". Proceedings of IEEE INFOCOM 2002, New York, NY, June 23-27 2002, pp.113119.

[18]. Y. Xu, J. Heidemann, D. Estrin. "Geography-informed Energy Conservation for Ad Hoc Routing". Proceedings of MobiCom'2001, Rome, Italy, July 2001, pp. 70-84.

[19]. B. Chen, K. Jamieson, H. Balakrishnan and R. Morris. Span: An Energy-Efficient Coordination Algorithm for Topology Maintenance in Ad Hoc Wireless Networks. Proceedings of MobiCom'2001, Rome, Italy, July 2001, pp. 85-96.

[20]. C. X. Mavromoustakis, H. D. Karatza, "Handling Delay Sensitive Contents using Adaptive Traffic-based Control Method for Minimizing Energy Consumption in Wireless Devices". Proceedings of 38th Annual Simulation Symposium (ANSS), IEEE Computer Society Press, SCS, San Diego, CA, April 2-8, 2005, pp. 295-302.

[21]. T. Janevski. "Traffic Analysis and Design of Wireless IP Networks" (Artech House Mobile Communications), ISBN: 1580533310, 2003 ARTECH House.

[22]. L. Feeney, M. Nilsson, "Investigating the energy consumption of a wireless network interface in an ad hoc networking environment". Proceedings of IEEE InfoCom, 5(8), 22-26 April 2001, vol.3, 2001, pp. 1548-1557.

[23]. C. X. Mavromoustakis, H. D. Karatza. "Segmented File Sharing with Recursive Epidemic Placement Policy for Reliability in Mobile Peer-to-Peer Devices". Proceedings of the 13th Annual Meeting of the IEEE International Symposium on Modeling, Analysis, and Simulation of Computer and Telecommunication Systems (MASCOTS), Georgia Tech, Atlanta, Georgia, September 26-29, 2005, pp 371-380. 